\title{
Mutation in Arabidopsis HIT1 locus causing heat and osmotic hypersensitivity
}

\author{
Shaw-Jye Wu ${ }^{1,2} *$, Robert D. Locy², Joe J. Shaw ${ }^{3}$, Joe H. Cherry², Narendra K. Singh ${ }^{2}$ \\ ${ }^{1}$ Plant Pathology Department, 349 Hutchison Hall, University of California, Davis, CA 95616, USA \\ 2 Department of Biological Sciences, Auburn University, 101 Life Sciences Building, Alabama 36849, USA \\ 3 Institute of Molecular Biology and Medicine, University of Scranton, Scranton, Pennsylvania 18510, USA
}

Accepted June 26, 2000

\section{Summary}

A mutant strain of Arabidopsis thaliana var. Columbia whose seedling development was 2 to $3^{\circ} \mathrm{C}$ more sensitive to high temperature inhibition than wild type was isolated. This mutant (hit 1, for heatintolerant) did not show any phenotypic change when grown at $22^{\circ} \mathrm{C}$. However, incubation at $37^{\circ} \mathrm{C}$ for 4 days was determined to be lethal for the mutant but not for wild-type plants. Leaves of wild type respond to high temperature by becoming erect, but by comparison leaves of the hit 1 mutant remain horizontal and show a slightly wilty appearance. Furthermore, seedling development in hit 1 mutants was more sensitive to osmotic stress imposed by exogenous mannitol ( $>300 \mathrm{mmol} / \mathrm{L}$ ). These data imply that HIT1 may be responsible for water status regulation by which plants survive heat stress. Genetic analysis shows that the hit 1 phenotype is conditioned by a single recessive, nuclear mutation, and the HIT1 locus was closely linked to the nga280 microsatellite marker on chromosome 1.

\section{Introduction}

The synthesis and accumulation of a variety of heat shock proteins (HSPs) that function as molecular chaperones are undoubtedly significant molecular events leading to the recovery from sublethal heat shocks and leading to the acquisition of tolerance to subsequent lethal heat shock (for review, see Vierling 1991, Schöffl et al. 1998). However, there is additional evidence that other molecular, biochemical, and physiological events beside the synthesis of HSPs also may play a role in tolerance to heat stress (Cherry et al. 1994, Nover 1994), and defining the genetic determinants of both

\footnotetext{
* E-mail corresponding author: jyewu@ucdavis.edu
}

HSP-mediated and/or alternative pathways has not yet significantly advanced. We have investigated the possibility of defining mutants that are more thermosensitive than wild type, in an attempt to define those genes important to basal thermotolerance in Arabidopsis thaliana.

Here we report the isolation of the first such heat intolerant mutant hit1. The hit 1 mutant was identified by looking for seedlings that were able to germinate but not able to mature at high temperature. Genetic analysis showed that hit 1 is a single, recessive nuclear mutation. Its cross sensitivity to osmotic stress and its inability to show erect leaves during heat stress suggest that the normal function of the mutated locus may be required to adjust the water status in plant, and this adjustment is crucial for plants to survive during heat stress. 


\section{Materials and Methods}

\section{Plant material and mutant isolation}

Arabidopsis thaliana (ecotype Colombia) carrying the homozygous genetic marker $\mathrm{g} / 1$ (glabra) is referred to as the wild type throughout this report. Both wild type and ethyl methanesulfonate (EMS)-mutagenized seeds were obtained from Lehle Seeds Inc. (Round Rock, TX). Plant growing conditions were as described unless specified (Wu et al. 1996).

EMS-mutagenized $\mathrm{M}_{2}$ seed were sown on agar plates and allowed to germinate at $30^{\circ} \mathrm{C}$ for 1 week. Seeds that failed to display aerial organs (opened, green cotyledons) were moved onto new agar plates and placed at $22^{\circ} \mathrm{C}$ for another 10 days. Seeds that could recover from previous heat stress and become fully-developed seedlings (putative mutants) were subsequently transferred to soil and grown to maturity. Heat sensitivity of the obtained $\mathrm{M}_{3}$ seeds was retested again by germination at both low $\left(22^{\circ} \mathrm{C}\right)$ and high $\left(30^{\circ} \mathrm{C}\right)$ temperature for one week. Those plants whose progeny demonstrated a germination rate equal to wild type at $22^{\circ} \mathrm{C}$ but lower than wild type at $30^{\circ} \mathrm{C}$ were considered true mutants, and backcrossed to wild type at least once before further study.

\section{Measurement of temperature and osmotic sensitives}

The temperature sensitivity of seedling development was determined by incubating Petri plates containing seeds at various temperature $\left(22-37^{\circ} \mathrm{C}\right)$. The seed germination rate was determined as the percentage of seeds showing visible protruding radicles, and the seedling maturation rate was determined as the percentage of seeds showing fully opened, green cotyledons after one week. Survival of matured seedlings upon heat stress was tested by transferring entire plates containing 10-day-old seedlings to higher temperature conditions $\left(30-37^{\circ} \mathrm{C}\right)$. A critical temperature and duration that could unambiguously discriminate mutant from wild type individuals was determined to be $37^{\circ} \mathrm{C}$ for four days. These conditions were used in subsequent genetic analysis.

For osmotic sensitivity measurement, seedling maturation rate was determined by sowing seeds on agar plates supplemented with various mannitol levels. The number of seedlings showing expanded cotyledons after 2 weeks incubation at $22^{\circ} \mathrm{C}$ was determined. For the root elongation assay, seeds were sown on mannitol-free plates which were placed vertically to allow the roots to grow on the agar surface. After 4 to 5 days incubation at $22^{\circ} \mathrm{C}$, seedlings with roots 1 to $1.5 \mathrm{~cm}$ in length were carefully transferred to plates containing various mannitol levels. Plates were again placed vertically to allow roots to continuously grow. The increase of root length was measured daily for up to 7 days. At least two replicated plates were used for each temperature and mannitol treatment.

\section{Genetic analysis}

Crosses between mutant and wild type were accomplished by transferring pollen from the mutant to the stigma of emasculated wild type flowers. The $F_{1}$ and $F_{2}$ plants were tested for their heat sensitivity by planting seeds on agar plates at $22^{\circ} \mathrm{C}$ for 10 days followed by treatment at $37^{\circ} \mathrm{C}$ for 4 days.

For mapping of the mutated locus, mutants in the Columbia $\mathrm{gl}-1$ background were crossed to plants of the Landsberg erecta back- ground. $F_{2}$ plants were self-pollinated to produce the $F_{3}$ generation for the survivability assay described above. DNA of individual mutant plants from the $F_{2}$ generation was isolated for gene-mapping using microsatellite markers (Bell and Ecker 1994) or using cleaved amplified polymorphic sequence (CAPS) markers (Konieczny and Ausubel 1993).

\section{Results and Discussion}

Upon imbibition, the development of an Arabidopsis seedling begins with the protrusion of the radicle (seed germination) followed by the expansion and greening of cotyledons (seedling maturation). Elevated temperatures interrupt this ongoing developmental process. On agar plates no reduction was found in either germination or maturation rates for wild-type Arabidopsis seeds at temperatures from $25^{\circ} \mathrm{C}$ up to $30^{\circ} \mathrm{C}$. However, while most seeds were still able to germinate, seedling maturation was completely arrested at $35^{\circ} \mathrm{C}$. Subsequent transfer of $35^{\circ} \mathrm{C}$-germinated seeds back to $25{ }^{\circ} \mathrm{C}$ permitted their normal development. Observing for seedlings unable to complete maturation at a high but normally permissive temperature, e.g. $30{ }^{\circ} \mathrm{C}$, allows temperature sensitive mutants to be screened.

About 20,000 EMS-mutagenized $M_{2}$ seeds were screened for heat hypersensitive mutants by examining their seedling development at $30^{\circ} \mathrm{C}$ for 7 days. One selected mutant, hit 1 , was later confirmed by retesting seedling development in the $\mathrm{M}_{3}$ generation. The heat sensitivity of hit1 mutant seedlings during development is shown in Figure 1. Germination of the hit 1 mutant was completely inhibited at $35^{\circ} \mathrm{C}$, while wild type germination was fully inhibited only at $37^{\circ} \mathrm{C}$ (Fig. $1 \mathrm{~A}$ ). For seedling maturation, the temperatures at which complete inhibition occurred was $32^{\circ} \mathrm{C}$ for hit 1 and $35^{\circ} \mathrm{C}$ for wild type (Fig. 1B). Thus, the hit1 mutant was 2 to $3^{\circ} \mathrm{C}$ more sensitive to heat inhibition than wild type during seed germination and seedling maturation.

Because heat sensitivity may depend on the stage of seedling development, we determined whether hit 1 was also hypersensitive to heat at the subsequent stages of seedling development. For 10-day-old plants, i.e. with one visible leaf pair, the hit 1 mutant was totally bleached after 4 days exposure to $37^{\circ} \mathrm{C}$ while wild-type seedlings remained green in color (Fig. 2). Subsequent transfer of plants from this stress treatment to room temperature allowed the growth of wild type, but not hit1 (data not shown). This treatment was subsequently employed for genetic analysis. Results indicated that hit 1 is a single, recessive, nuclear mutation (Table 1) and the HIT1 locus was mapped to chromosome 1 closely linked (1.1\% recombination frequency) to nga280 marker (Table 2 ).

When mature plants, with 6-8 rosette leaves, were exposed to $37^{\circ} \mathrm{C}$, leaves of wild-type plants lifted upwards to an erect position whereas hit 1 leaves failed to do so (Fig. 3). Maximum erectness of wild-type leaves was reached within 24 hours after the initiation of treatment and was maintained 


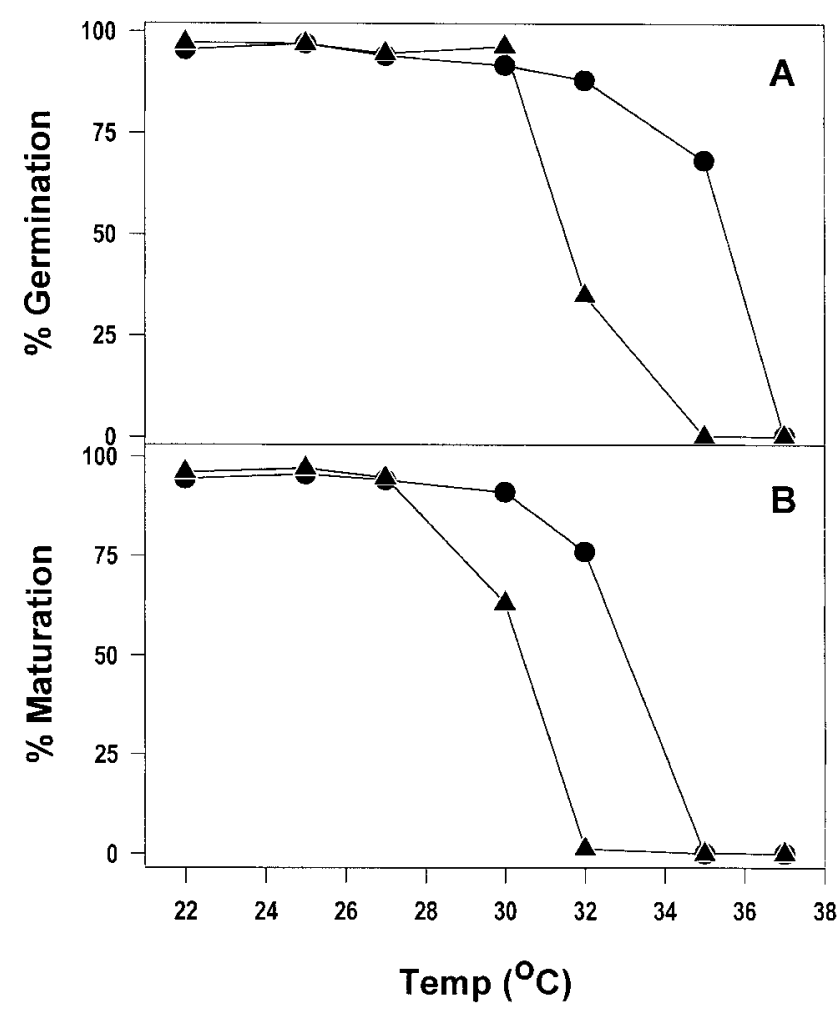

Figure 1. Seedling development of the hit 1 mutant is $2 \sim 3{ }^{\circ} \mathrm{C}$ more sensitive to heat inhibition than wild-type seedlings. Seeds were sown on agar plates and incubated at various temperatures. Percentage of germination (A) was determined as the percentage of seed that showed radical emergence, and percentage of maturation (B) was determined as the percentage of seedlings that showed opened cotyledons within one week. Data are obtained from a sample size of $\sim 100$ seeds at each temperature tested. $\bullet$, wild type; $\boldsymbol{\Lambda}$, hit1.

as long as the plants remained at the high temperature. Maintaining erect leaves in herbaceous plants may rely on the buildup of turgor pressure, particularly in cells of leaf bases. This implies the establishment of a new water status within the aerial portions of the wild-type plants, and prompts us to further examine the water-regulating capability of the hit 1 mutant upon osmotic stress.

As shown in Figure 4A, none of the hit 1 seeds were able to develop into mature seedlings when the concentration of mannitol was above $300 \mathrm{mmol} / \mathrm{L}$. For wild-type seeds, a mannitol concentration for complete inhibition of seedling development occurred at greater than $400 \mathrm{mmol} / \mathrm{L}$. Although hit 1 seeds failed to develop into mature seedlings on high mannitol media $(300-400 \mathrm{mmol} / \mathrm{L})$, they were still able to germinate. Figure $4 \mathrm{~B}$ shows that there is little difference in root growth between wild type and hit 1 at high mannitol concentrations. This result suggests that the osmotic hypersensitivity of the hit 1 mutant may involve water relation only in the aerial portion but not in the root of the plant.

Functional genetics involving isolation and characterization of hypersensitive mutants, has provided new insight into plant responses to cold, salt, as well as heavy metals (Hugly et al. 1990, Howden and Cobbett 1992, Wu et al. 1996, Liu and Zhu 1997, Liu and Zhu 1998). Nonetheless, using such a genetic approach to study plant responses to heat stress has been limited. One reason for this is that mutants resulting in changes in the thermal stability of any protein involved in basic cellular functions such as metabolism could potentially generate a temperature sensitive phenotype. However, the hit1 mutant presented here does not support this assumption since the heat-hypersensitivity always cosegregates with the osmotic hypersensitive phenotype which requires no involvement of temperature. The heat hypersensitivity of the hit $1 \mathrm{mu}-$ tant is also unlikely to be in defect of transpirational cooling because seed germination and seedling development are also inhibited by high temperature. Rather, the defect of the hit1 mutant most likely lies in the mechanism for regulating the water status, especially the aerial portion of the plant, that is essential for plant survival and development during heat and osmotic stresses. Results from a recent study in which enhanced thermotolerance in both seed germination and seed-

Table 1. Genetic Analysis of Arabidopsis hit1 mutant.

\begin{tabular}{|c|c|c|c|c|c|}
\hline $\begin{array}{l}\text { Strains or Crosses } \\
\left(\propto \times O^{\wedge}\right)\end{array}$ & Generation & Total & Tolerant $^{a}$ & Sensitive $^{a}$ & $\chi^{2 b}$ \\
\hline Wild-type Columbia & & 551 & 551 & 0 & \\
\hline hit1/hit 1 & & 520 & 0 & 520 & \\
\hline \multirow[t]{2}{*}{ Wild-type $\times$ hit 1/hit 1} & $F_{1}$ & 37 & 37 & 0 & \\
\hline & $\mathrm{F}_{2}$ & 530 & 412 & $\begin{array}{l}118 \\
(22.3 \%)\end{array}$ & $2.097^{b}$ \\
\hline \multicolumn{6}{|c|}{$\begin{array}{l}\text { Tolerant or sensitive was determined by viability of } 10 \text {-day-old seed- } \\
\text { lings at } 37^{\circ} \mathrm{C} \text { for } 5 \text { days. } \\
\text { b The calculated } \chi^{2} \text { value was based on the expected ratio of } 3: 1 \text { for } \\
T: S \text { individuals, assuming that hit } 1 \text { was a single recessive mutation } \\
(P>0.05) \text {. }\end{array}$} \\
\hline
\end{tabular}

Table 2. Genetic Mapping of hit 1.

\begin{tabular}{lllc}
\hline Marker & Chromosome & $\mathrm{n}^{\mathrm{a}}$ & Recombination Frequency $^{\text {b }}$ \\
\hline nga 63 & I & 40 & $50.0 \pm 5.6$ \\
nga 280 & I & 44 & $1.1 \pm 1.1$ \\
GPA 1 & II & 35 & $37.1 \pm 5.8$ \\
nga 168 & II & 35 & $40.0 \pm 5.9$ \\
nga 126 & III & 44 & $59.0 \pm 5.2$ \\
nga 8 & IV & 44 & $39.8 \pm 5.2$ \\
AG 4 & IV & 44 & $40.9 \pm 5.2$ \\
nga 76 & V & 34 & $51.5 \pm 6.1$ \\
nga 225 & V & 30 & $50.0 \pm 6.5$ \\
\hline
\end{tabular}

a Number of individual $F_{2}$ analyzed.

b Calculated by the Kosambi function (Koornneef and Stam, 1992). 


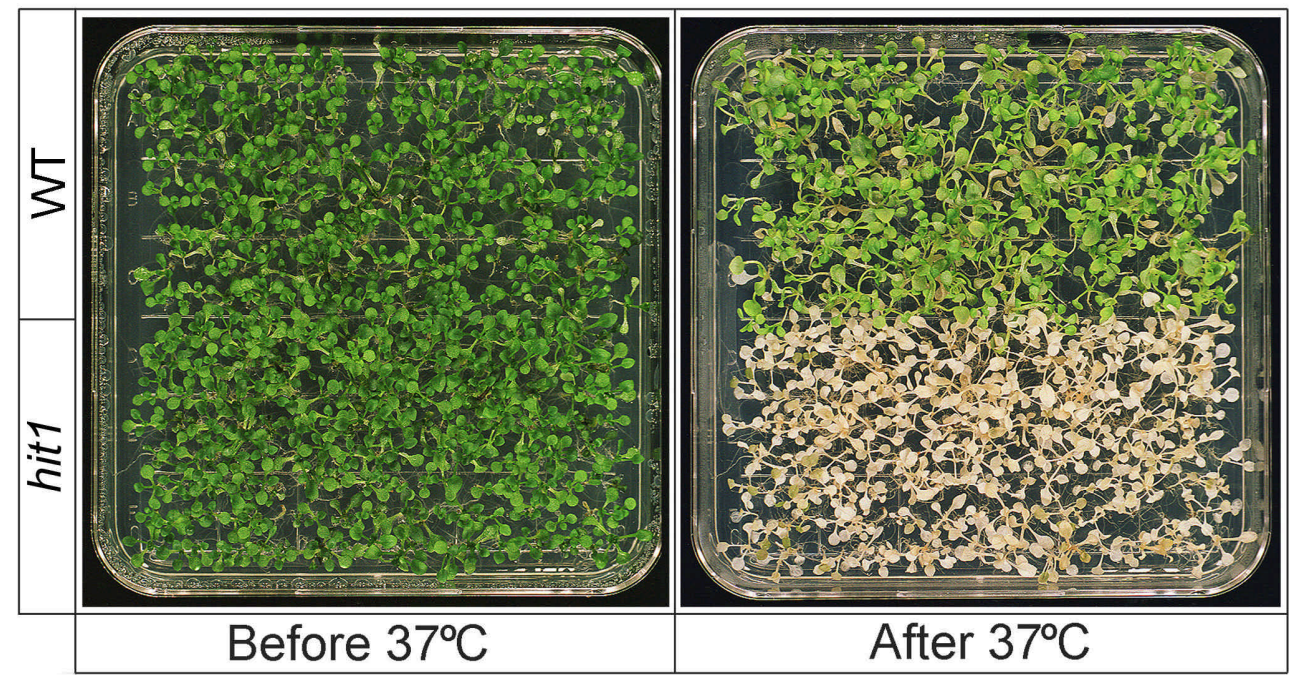

Figure 2. Incubation at $37^{\circ} \mathrm{C}$ for 4 days was lethal to hit 1 but not wild type. Ten-day-old media-grown plants, with the first pair of leaves, were transferred to $37^{\circ} \mathrm{C}$ and incubated for four days. WT, wild type.

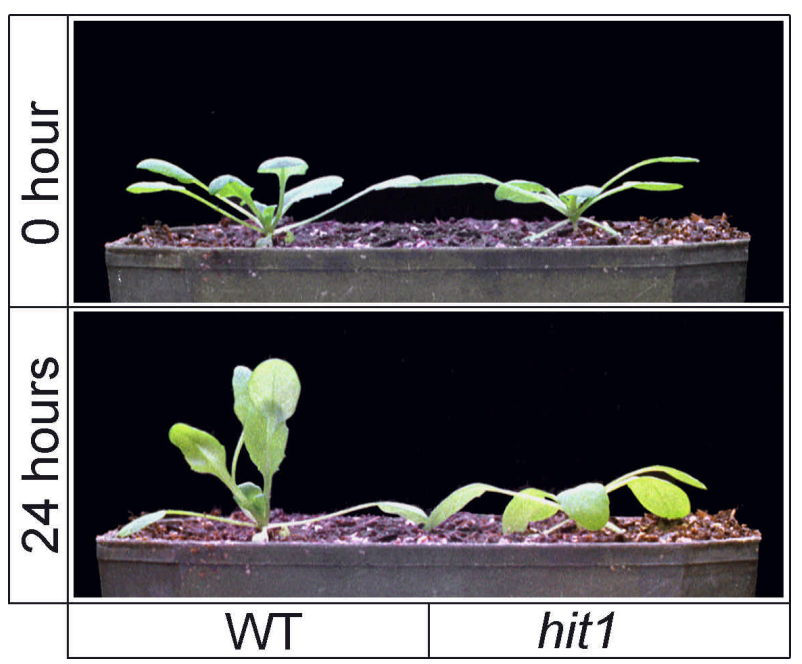

Figure 3. Soil-grown hit1 plants failed to lift their leaves upwards to an erect position as did wild-type plants. Plants with $6-8$ rosette leaves were transferred to $37^{\circ} \mathrm{C}$ and incubated for 24 hours.

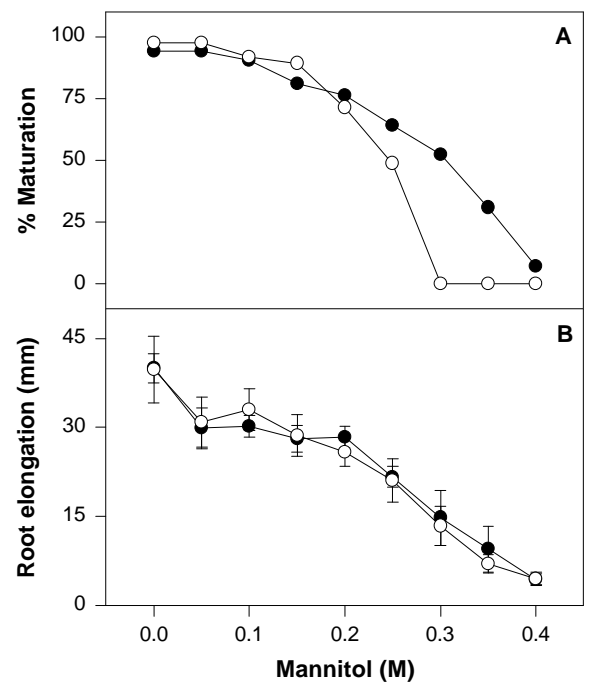

Figure 4. Seedling development of hit1 was more sensitive to osmotic stress inhibition. (A) Seeds were sown on agar plates containing various concentrations of mannitol as an osmoticum. The percentage of seedling maturation was calculated from the number of seedlings that showed green, opened cotyledons within two weeks from the beginning of the experiment. Data are obtained from a sample size of $\sim 100$ seeds at each temperature tested. (B) Root elongation of hit1 and wild type seedlings was measured to quantify sensitivity toward osmotic stress. Root elongation data 7 days after transfer to mannitol containing plates is shown. 0 , wild type; $\bigcirc$, hit1. Each point represents the means $\pm \operatorname{SE}(n=10)$. 
ling maturation was achieved by overproduction of glycinebetaine support such a notion (Alia et al. 1998).

In summary, the isolation of the hit 1 mutant demonstrates the feasibility of a molecular genetic approach to study plant heat stress responses. The hit 1 mutant is valuable for understanding the mechanisms by which plants cope with heat stress at both the molecular and whole plant levels. The cross sensitivity of hit 1 to osmotic stress also provides us with the opportunity to examine the interaction between thermal and osmotic stress responses.

Acknowledgements. S.-J. Wu would like to thank to Dr. Jian-Kang Zhu (Department of Plant Sciences, University of Arizona) for helpful advice during the initial inspiration of this work. Support for this study by BARD Grant (US-2743-96) is greatly acknowledged.

\section{References}

Alia HH, Sakamoto A, Murata N (1998) Enhancement of the tolerance of Arabidopsis to high temperatures by genetic engineering of the synthesis of glycinebetain. Plant J 16: 155-161

Bell CJ, Ecker JR (1994) Assignment of 30 microsatellite loci to the linkage map of Arabidopsis. Genomics 19: 137-144

Cherry JH, Mayer RR, Heuss-LaRosa K, Reddy PM, Singh NK (1994) Altered gene expression in thermoadapted cultured cells of cow- pea. In: Cherry JH (ed) Biochemical and Cellular Mechanisms of Stress Tolerance in Plants. Springer-Verlag, Berlin, pp 229-242

Howden R, Cobbett CS (1992) Cadmium-sensitive mutants of Arabidopsis thaliana. Plant Physiol 99: 100-107

Hugly S, McCourt P, Browse J, Patterson GW, Somerville C (1990) A chilling sensitive mutant of Arabidopsis with altered steryl-ester metabolism. Plant Physiol 93: 1053-1062

Konieczny A, Ausubel F (1993) A procedure for quick mapping of Arabidopsis mutants using ecotype specific markers. Plant $\mathrm{J} 4$ 403-410

Koornneef M, Stam P (1992) Genetic analysis. In: Koncz C, Chua NH, Schell J (eds) Methods In Arabidopsis Research. World Scientific, Singapore, pp 83-99

Liu J, Zhu JK (1997) An Arabidopsis mutant that requires increased calcium for potassium nutrition and salt tolerance. Proc Natl Acad Sci (USA) 94: 14960-14964

Liu J, Zhu JK (1998) A calcium sensor homolog required for plant salt tolerance. Science 280: 1943-1945

Nover $L$ (1994) The heat stress response as part of the plant stress network. In: Cherry JH (ed) Biochemical and Cellular Mechanisms of Stress Tolerance in Plants. Springer-Verlag, Berlin, pp 3-45

Schöffl F, Prändl R, Reindl A (1998) Regulation of the Heat-shock Response. Plant Physiol 117: 1135-1141

Vierling E (1991) The roles of heat shock proteins in plants. Annu Rev Plant Physiol Mol Biol 42: 579-620

Wu SJ, Ding L, Zhu JK (1996) SOS1, a genetic locus essential for salt tolerance and potassium acquisition. Plant Cell 8: 617-627 